\title{
Fuzzy modelling and efficiency in health care systems
}

\author{
Ahmet F. Ozok \\ Industrial Engineering Department, Istanbul Kultur University, Atakoy Kampusu, İstanbul, TURKEY
}

\begin{abstract}
American Medical Institute reports that each year, because of the medical error, minimum fifty thousand people are dead. For a safety and quality medical system, it is important that information systems are used in health care systems. Health information applications help us to reduce the human error and to support patient care systems. Recently, it is reported that medical information systems applications have also some negative effect on all medical integral elements. The cost of health care information systems is about $4.6 \%$ of the total cost. In this paper, it is tried a risk determination model according to principles of fuzzy logic. The improvement of health care systems has become a very popular topic in Turkey recent years. Using necessary information system; it became possible to care patients in a safer way. However, using the necessary HIS tools to manage of administrative and clinical processes at hospitals became more important than before. For example; clinical work flows and communication among pharmacists, nurses and physicians are still not enough investigated. We use fuzzy modeling as a research strategy and developed sum fuzzy membership functions to minimize human error. In application in Turkey the results are significantly related with each other. Besides, the sign differences in health care information systems strongly effects of risk magnitude. The obtained results are discussed and some comments are added.
\end{abstract}

Keywords: Health care system, fuzzy modeling, health care information system, improvement of health care systems, system design

\section{Introduction}

As it is mentioned in abstract, approximately 50.000 people are dead because of medical errors in United States. This amount is more than because of traffic accidents, breath cancer and aids. One of the most important problems in health care systems is to accumulate clinical, administrative and financial data. If we have a sound storage then we can have feedback to deductive reasoning. Health care systems in medical systems give us the opportunity to reduce all kind of errors and to support all human parts of patient care systems. (Bates et al., 2001; Amenwerth et al., 2003) On the other side implementing information system in health care systems has also some negative effects (Patterson et al., 2002; Koppel et al., 2008) It means that in application we have to eliminate them. The cost of application of health care information systems is about $4.6 \%$. In this paper it is tried to determine the magnitude of risk before the implementation. Using this pro-active estimation model it is possible to reduce the risk and to take necessary measurements. Before defining the factors of the model, we tried to define the necessary elements of the model. It is extremely important (like in aviation, nuclear stations and nitration industry) that the critical processes of the patients have to be analyzed and to prevent the risky results. We have to accept that although taking the necessary measurements there are also some risk factors which cannot be prevented. But to our mind it has not to be larger than $1 \%$. In risk evaluation systems, we used "Fault Tree Analysis", "Even Try Analysis" and "Failure Mode and Effect Analysis. "In Even Tree Analysis, we have to have the probability of success and failure. In many cases it is very difficult to find the risk probability functions. This is especially important in "Fault Tree Analysis." Fault Tree Analysis is suitable to analyze the past data. On the other side in "Failure Mode and Effect Analysis", 
it is possible to define potential error. Because of all these reasons to our mind Electronic Failure Mode and Effect Analysis is the most suitable method in HIS. In practice, it is known that there are many factors in risk assessment. But they are not taken in consideration. In our model, the weight of potential risk factors is determined according to "Analytical Network Process." In this model, all the factors which effect to decision making are taken in a
Hierarchal Level. In the model we assumed that the factors in the same level are independent from each other. For this reason to determine the weight of factors we used "Analytical Network Process" (ANP). As it is known in this method all dependences among the factors are considered. We assume that the risk magnitude becomes greater if the new technology is more different than classical methods.

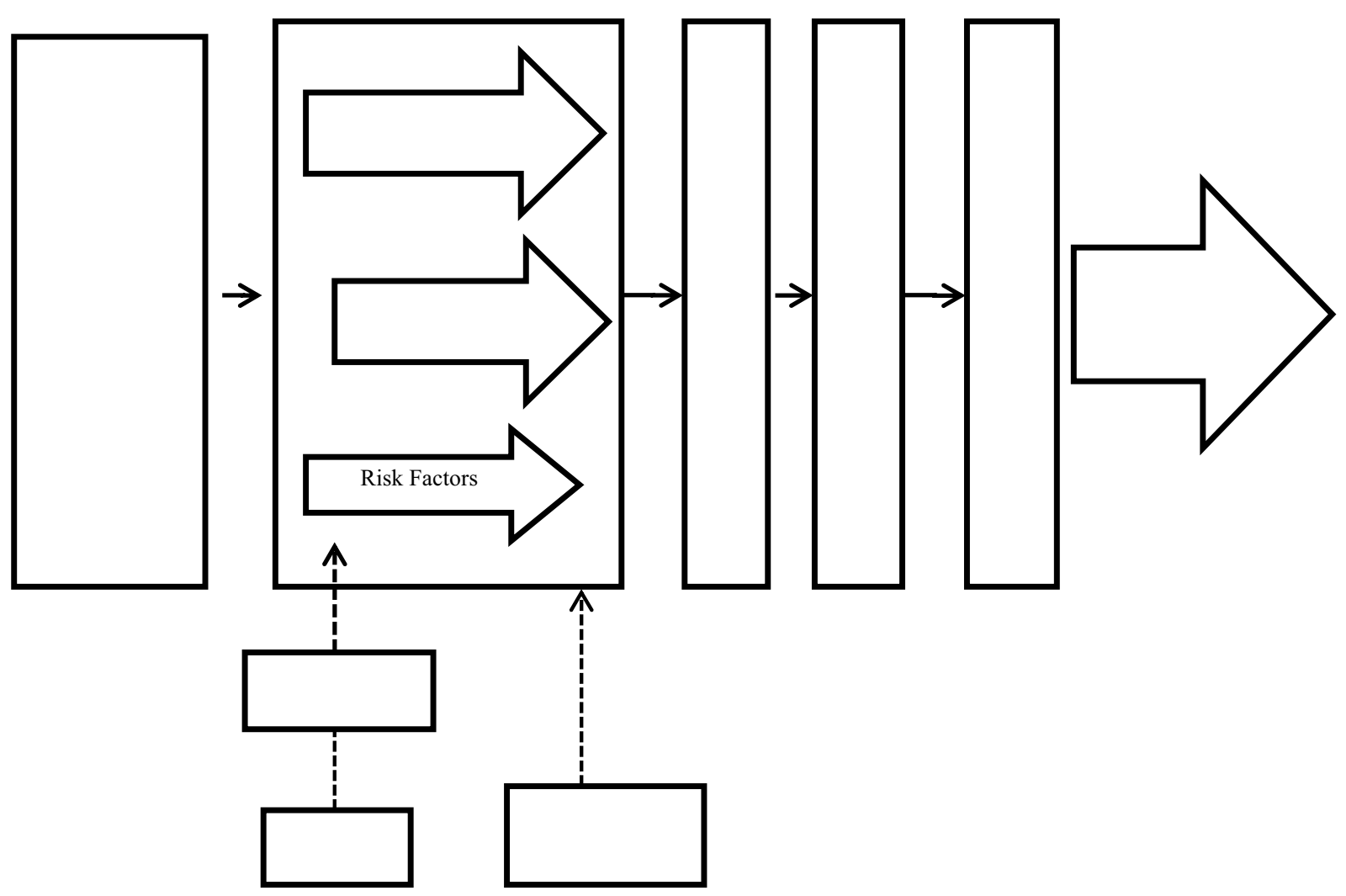

Figure 1

The structure of the health care system evaluation method (Gulcin et al., 2011)

For this reason it is important to determine the significant differences between old and new methods. Fuzzy Modeling is especially successful if we have many factors and they are related with each other in a very complex way. We use expert decisions as low, high, and very low. In Reliability Analysis we used Linguistic Terms. We tried to create a new risk determining model.

\section{Method}

The different steps have to be taken in risk model. According to these steps first of all we had to establish an expert group and then to define risk factors. According to ANP Method the weight of each factor is determined according to expert judgments. The experts determine the probability of 
risk, the height of the risk and the importance of each variables. And then linguistic variables are transformed to standard fuzzy numbers and combined with expert judgments. And then we obtained fuzzy numbers and using fuzzy inference we obtained the risk values.

\subsection{The Necessary Steps for Health System Evaluation}

Risk determination groups are composed from experts who came from different areas. The weight of each expert is determined according the following formula (Cebi and Kahraman, 2010);

$$
W e i=\frac{P e i}{\sum_{i=1}^{Z} P e i}
$$

Risk factors which can have an impact on the performance of HIS are determined by surveys, literature or any group which focuses on risk factors. To determine importance of each factor is determined according to linguistic scale for ANP:

Table 1: Linguistic scale for ANP

\begin{tabular}{cl}
\hline Importance & Definition \\
\hline 1 & Equally important \\
3 & Weakly more important \\
5 & Strongly more important \\
7 & Very strongly more important \\
9 & Extremely more important \\
\hline
\end{tabular}

Factor index, risk likelihood and risk severity are determined according to following formulas;

$$
\begin{aligned}
& \tilde{P} i=\left(\tilde{P} i^{1} x W_{e 1}\right)+\left(\tilde{P} i^{2} x W_{e 2}\right)+\cdots \\
& +\left(\tilde{P} i^{m} x W_{e m}\right) \\
& F I=\sum_{i=1}^{n} W i P i \\
& R L=\left(R \widetilde{L^{1}} x W_{e 1}\right)+\left(R \widetilde{L^{2}} x W_{e 2}\right)+\cdots \\
& +\left(R \widetilde{L^{m}} \times W_{\text {em }}\right) \\
& R L=\frac{\sum_{i=1}^{n} R L_{i}}{n} \\
& R \tilde{S}=\left(R \widetilde{S_{1}} x W_{e 1}\right)+\left(R \widetilde{S_{2}} x W_{e 2}\right)+\cdots \\
& +\left(R \widetilde{S_{m}} x W_{\text {em }}\right)
\end{aligned}
$$

The aggregated fuzzy numbers of FI, RL, and RS are converted into fuzzy sets. The fuzzy inference system is very suitable to combine subjectivities, factor compared with the others using linguistic uncertainties and imprecise and vogue information in complex systems. One can use "if then" rules to formulate the conditional statements.

\section{Implementation of Fuzzy Modeling in a Hospital}

As it is mentioned before, health care systems become more and more important to improve all over the world and also in Turkey. One can claim that implementing information technologies in health care systems can increase the efficiency of patient safety from $60 \%$ to $90 \%$. In the hospital the efficiency of health care systems is mainly dependent on the following factors;

- Age of participated people

- Gender

- Educational Level

- Skills

- Individual Efficiency

- Experience

- Training

- Communication 1

- Communication 2

- Organizational Commitment

- User Involvement

- Number of the people

- Coordination Level 1

- Coordination Level 2

- Ease of use the system

- Usefulness

- Compatibility

- Impact

- Enjoyment

- General psychological factors

\section{Conclusion}

In application if we combine risk level and risk severity, one can classify the risk magnitude as negligible, minor, major and critical. According to each, level one has to take industrial engineering measurements in a scientific and detailed way. As an application case, the health care system in Turkey is a transitional phase. The legislative rules of health care person are not still clear in every detail. For the time being the scientific environment efforts are strengthened and still continuously change. At the 
research level obtained results seems to be improved. Because of the characters of the problem mathematical modeling of health care systems is clearly more suitable for fuzzy modeling. We have noticed at the end that different hospitals have different organizational character and one has to take into consideration of these properties. It is also possible, as some research reveals that negative effect of health IT applications can also occur. In any case all risk factors have to be considered and new technology negative effects have to be eliminated. Using fuzzy modeling, we can be able to process experts' evaluations to make imprecise and vogue information into account, to integrate possible risk factors into decision making process and to obtain risk likelihood. To proceed ANP together with Fuzzy Logic relative importance of each factor can be obtained. The gap between ideal model and reality can also be handled.

In the future using a system approach in industrial engineering all factors (even building technical facilities, personal characteristics, job requirements, social psychosocial and cultural factors etc.) which effect on health care systems has to be considered and we hope that fuzzy modeling applications in the future will gain much more importance.

\section{References}

[1] Bates, D.W., Cohen, M., Leape, L. L., Overhage, J. M., Shabot, M. M., \& Sheridan, T. (2001). Reducing the frequency of errors in medicine using information technology. JAMIA, 8(4), 299-308.

[2] Amenwerth, E., Graber, S., Hermann, G., Bürkle, T., \& König, J. (2003). Evaluation of health information systemsproblems and challenges. International Journal of Medical Informatics 71, 125-135.

[3] Patterson, E. S., Cook, R. I., \& Render, M. L. (2002). Improving patient safety by identifying side effects from introducing barcoding in medication administration. Journal of the American Medical Informatics Association, 9(5), 540-553.

[4] Koppel, R., Wetterneck. T., Telles, J. L. \& Karsh, B. T. (2008). Workarounds to barcode medication administration systems: Their occurrences causes and threats to patient safety. Journal of the American Medical Informatics Association, 15, 408-423.

[5] Cebi, S., \& Kahraman, C. (2010). Developing a group decision support system based on fuzzy information axiom. Knowledge-Based System, 23(1), 3-16.

[6] Yucel, G., Cebi, S., Hoege, B., \& Ozok, A. F., (2011). A fuzzy risk assessment model for hospital information system implementation. 